\title{
Combination of Pigment Epithelium Derived Factor (PEDF) With Anti- Vascular Endothelial Growth Factor (VEGF) Therapy Protects the Retina From Ischemic Damage
}

Lei Xi ( $\square$ everydayxilei@163.com )

University of Tübingen

Research

Keywords: Ischemia, Pigment epithelium derived factor, Retina, Vascular endothelial growth factor

Posted Date: August 19th, 2021

DOl: https://doi.org/10.21203/rs.3.rs-814635/v1

License: (c) (1) This work is licensed under a Creative Commons Attribution 4.0 International License. Read Full License 


\section{Abstract}

\section{Background}

Ocular ischemia is a vision-threatening disease, and is a medical condition associated with many ocular diseases. Anti-vascular endothelial growth factor (VEGF) therapy successfully prevents the progression of retinal neovascularization caused by ocular ischemia. Anti-VEGF therapy has limitations related to its side effects and suppression of physiological revascularization. Pigment epithelium derived factor (PEDF) has anti-angiogenesis and neurotrophic neuroprotective functions. It is a promising agent in the treatment of ischemia-induced retinal neurodegeneration. The purpose of this study is to investigate the effect of PEDF and anti-VEGF and the combined therapy on the ischemic rat eye model ex vivo.

Methods

We have previously established an ex vivo model of rat ocular ischemia by incubating the freshly enucleated eye in Dulbecco's modified eagle medium (DMEM) at $4{ }^{\circ} \mathrm{C}$ for 14 hours. This ex vivo eye model has already been confirmed as an ocular ischemia model. In this study, 36 eyes of 18 rats were used and each group included 6 eyes. The PEDF protein $(10 \mu \mathrm{g}, 4 \mu \mathrm{l})$, anti-VEGF drug (Avastin, $50 \mu \mathrm{g}, 4 \mu \mathrm{l})$ or combined PEDF/Avastin (10 $\mu$ g PEDF $+50 \mu \mathrm{g}$ Avastin, $4 \mu \mathrm{l})$ were intravitreally injected after eye enucleation. Fourteen hours later, eyes were fixed by formalin and immunohistochemical staining for VEGF, PEDF and glial fibrillary acidic protein (GFAP) was performed. The immunohistochemical staining intensity was analyzed for each group. Tunnel staining was used to determine the apoptosis of retinal neural cells.

Results

The immunohistochemical staining intensity of VEGF and GFAP was significantly reduced in the neuroretina, the RPE cell layer and the choroidal vessels of the double treatment (PEDF/Anti-VEGF) eyes compared to the vehicle, PEDF, and anti-VEGF treatment alone. The intravitreally injected PEDF protein can penetrate the retina and locate in the neural retina, the RPE layer and the choroidal vessels. Compared to the vehicle-treated group, both the PEDF-treated and the double-treated groups showed significantly decreased number of TUNEL-positive nuclei, and the PEDF/Anti-VEGF treatment group had the least TUNEL-positive nuclei.

Conclusions

PEDF/Anti-VEGF therapy shows a synergistic effect in the treatment of ischemic eye diseases. The combination of PEDF and anti-VEGF drug (Avastin) is a possible therapeutic strategy against ischemic retinal and choroidal diseases.

\section{Background}


Retinal ischemia is a medical condition associated with many ocular diseases, such as branch retinal vein occlusion (BRVO), acute angle-closure glaucoma, retinal vascular occlusions, diabetic retinopathy, and retinopathy of prematurity. These diseases can lead to visual impairment and even blindness in severe cases. Ischemic retinopathies are characterized by retinal vasculature impairment and local hypoxia, which trigger an exaggerated and uncontrollable pathological neovascularization (NV) [1]. AntiVEGF therapy has proved effective in reducing the NV areas of some patients with retinopathy. However, anti-VEGF agents have limited benefits, they do not facilitate revascularization of the retina, resulting in adverse outcomes [2]. Furthermore, VEGF and other biological mediators play an important role in physiological angiogenesis [3]. Anti-VEGF therapy is effective in inhibiting VEGF and reversing neovascularization, it also inhibits physiological revascularization $[4,5,6]$. Several other treatments such as retinal laser photocoagulation, transpupillary thermotherapy and photodynamic therapy are used to treat ischemia-related retinal diseases, but these approaches are damaging to the retina and not always effective $[7,8]$.

Retinal neurons, such as retinal photoreceptor cells and retinal ganglion cells (RGCs) are responsible for the transmission of the visual information process to the brain and are vulnerable to ischemia [9]. To date, there are no clinically approved drugs that can effectively protect retinal neurons from ischemic injury. It is necessary to develop new therapies to treat ischemic retinopathy and protect retinal neurons from apoptosis. Pigment epithelium-derived factor (PEDF) was first identified from conditioned medium of human retinal pigment epithelium as a neuronal differentiating factor [10] and then found to be a potential anti-angiogenic factor [11], with neurotrophic and neuroprotective functions [12]. PEDF is a multifunctional factor which promotes RGC and photoreceptor cell survival $[13,14]$, inhibits pathologically increased vascular permeability [15] and downregulates VEGF expression [16]. PEDF may be a potential anti-angiogenesis and neuroprotective factor for the treatment of ischemic retinal diseases.

Retinal tissue ischemia or hypoxia are pathogenic determinants in ocular diseases and the ischemic condition stimulates an upregulated expression and release of vascular endothelial growth factor (VEGF) [17] and a downregulation of the PEDF level [18]. The unbalanced level of VEGF/PEDF may induce retinal neovascularization and other disorders. In the present study, we aimed to use an already established ischemic eye model to investigate the effect of intravitreal injection of PEDF or PEDF combined with an anti-VEGF drug (Avastin) in the ischemic retina and choroid ex vivo. We also examined the distribution and expression of vascular endothelial growth factor (VEGF) and glial fibrillary acidic protein (GFAP) in the retina or the choroid. The results showed that PEDF combined with anti-VEGF can protect the RGCs from ischemic retinopathy and suppress VEGF and GFAP in the retina ex vivo.

\section{Materials And Methods}

\section{Experimental animals}

Adult Long Evans rats $(n=18)$ weighing $250-300 \mathrm{~g}$ were used in this study. All animals were handled in accordance with the German Animal Welfare Act and were under the control of the animal protection 
agency and supervision of veterinarians of the University of Tübingen. The rats had free access to water and food in the animal facility. All the rats were euthanized with carbon dioxide immediately before enucleation of the eyes.

\section{Experimental design}

The ischemic rat eye model was established by incubating the freshly enucleated eye in a tube $(50 \mathrm{~mL})$ filled with Dulbecco's modified eagle medium (DMEM) $(25 \mathrm{~mL})$ at $4^{\circ} \mathrm{C}$ for 14 hours. This ischemic eye model had already been established by us. We had six separate groups in this experiment and each group included six eyes. Group 1 was the control group, the rat eye was enucleated and immediately fixed by formalin; Group 2 was the 14-hour ischemia/hypoxia alone group, the rat eye was enucleated and incubated in the DMEM solution at $4{ }^{\circ} \mathrm{C}$ for 14 hours; Group 3 was the vehicle-treated group, the rat eye was enucleated, injected with vehicle intravitreally (PBS, $4 \mu \mathrm{l}$ ) and incubated in the DMEM solution at $4^{\circ} \mathrm{C}$ for 14 hours; Group 4 was the PEDF-treated group, the rat eye was enucleated, injected with PEDF protein intravitreally $(10 \mu \mathrm{g}, 4 \mu \mathrm{l})$ and incubated in the DMEM solution at $4^{\circ} \mathrm{C}$ for 14 hours; Group 5 was the Avastin-treated group, the rat eye was enucleated, injected Avastin intravitreally $(50 \mu \mathrm{g}, 4 \mu \mathrm{l})$ and incubated in the DMEM solution at $4^{\circ} \mathrm{C}$ for 14 hours; Group 6 was the PEDF/Avastin treatment group, the rat eye was enucleated, injected with PEDF protein and Avastin intravitreally $(10 \mu \mathrm{g}$ PEDF $+50 \mu \mathrm{g}$ Avastin, $4 \mu \mathrm{l})$ and incubated in the DMEM solution at $4^{\circ} \mathrm{C}$ for 14 hours. Finally, all the eyes were fixed by $4.5 \%$ formalin (Roti Histofix, Carl Roth, Karlsruhe, Germany) and embedded in paraffin for histologic study.

\section{Intravitreal injection}

$4 \mu \mathrm{l}$ solution (PBS, $10 \mu \mathrm{g}$ PEDF protein, $50 \mu \mathrm{g}$ bevacizumab, both $10 \mu \mathrm{g}$ PEDF protein and $50 \mu \mathrm{g}$ bevacizumab) was delivered into the vitreous cavity by using a NanoFil 34-gauge bevelled needle (Hamilton Co., Reno, NV, USA).

\section{Immunohistochemistry}

Sections $4 \mu$ m-thick were cut from paraffin embedded samples. The sections were dewaxed in xylene, rehydrated in ethanol, and boiled at high pressure in citrate buffer $(\mathrm{pH} 6.0)$ solution for 2 minutes for antigen retrieval and then taken out and stained with respective primary antibodies: VEGF (1:50, Gene Tex, USA), GFAP (1:50, CST, \#3670, MA, US) and human PEDF (1:500, Abcam, ab233120). After incubation for 30 minutes with the primary antibody at $37^{\circ} \mathrm{C}$, a thorough wash with $1 \times$ Tris-buffered saline (TBS; Merck, Darmstadt, Germany) $(3 \times 5 \mathrm{~min})$ was performed. ImmPRESS Poly-AP Anti-Rabbit (MP5401; Vector laboratories, Peterborough, UK) antibody or ImmPRESS Poly-AP Anti-mouse (MP5402; Vector laboratories, Peterborough, UK) antibody was applied for 30 minutes at room temperature. After another washing, the sections were counterstained with hematoxylin (Carl Roth, Karlsruhe, Germany) for 25 seconds and washed completely with tap water. Finally, the slides were rehydrated and dehydrated, then dried and cover-slipped.

The immunohistochemical staining intensity was analyzed and quantified. The staining intensity is graded from 0 to 4 . Grade 0 is completely negative and grade 4 is the most strongly stained. The 
schematic diagram of the staining intensity is shown in Fig. 1 (neural retina, RPE layer and choroidal vessel). For each eye, the staining intensity was evaluated for the whole neural retina, RPE and choroid under light microscopy at a magnification of $40 \times$. The width of each field of view is about $350 \mu \mathrm{m}$. From one end of the retina to the other end of retina, each field of view is given a grade according to the staining intensity. The number of visual fields evaluated for each group was shown in Table 1.

Table 1

The number of visual fields evaluated for different primary antibody-staining in each group

\begin{tabular}{|lllllll|}
\hline Antibody & Group 1 & Group 2 & Group 3 & Group 4 & Group 5 & Group 6 \\
\hline VEGF-neural retina & 94 & 83 & 108 & 96 & 94 & 95 \\
\hline VEGF-RPE & 82 & 94 & 113 & 103 & 108 & 99 \\
\hline VEGF-choroid & 84 & 97 & 109 & 102 & 110 & 98 \\
\hline GFAP & 83 & 88 & 91 & 86 & 81 & 84 \\
\hline PEDF-RPE & 44 & 49 & 59 & 57 & 57 & 39 \\
\hline PEDF-choroid & 40 & 50 & 59 & 59 & 59 & 38 \\
\hline
\end{tabular}

VEGF: vascular endothelial growth factor; RPE: retinal pigmented epithelium; GFAP: glial fibrillary acidic protein

\section{Terminal Deoxynucleotidyl Transferase (TdT)-Mediated dUTP Nick End Labeling (TUNEL) Assay}

Cell death was assessed using the TUNEL assay by means of an in situ cell death detection TMR red TUNEL kit (Roche Diagnostics, Mannheim, Germany). The paraffin sections were deparaffinized. After washing in $0.1 \mathrm{M}$ phosphate buffer saline (PBS), the samples were incubated in Proteinase $\mathrm{K}$ at $37^{\circ} \mathrm{C}$ for $5 \mathrm{~min}$ for cell permeabilization. Then the sections were incubated with alcohol acetic acid mixture $(62 \%$ $\mathrm{EtOH}, 11 \%$ Acetic Acid, 27\% $\mathrm{H}_{2} \mathrm{O}$ ) for $5 \mathrm{~min}$, and blocked for 1 hour. Finally, samples were incubated in terminal deoxynucleotidyl transferase enzyme at $37^{\circ} \mathrm{C}$ for $60 \mathrm{~min}$ in the dark and washed two times by PBS. Nuclei were counterstained with 4',6-diamidino-2-phenylindole (DAPI). The slides were photographed with an Imager Z2 ApoTome microscope (Carl Zeiss Microscopy GmbH, Oberkochen, Germany). The percentage of TUNEL-positive nuclei to the total number of ganglion cells per $100 \mu \mathrm{m}$ length of the retina (TUNEL-positive nuclei/total ganglion nuclei $\times 100$ ) and the number of TUNEL-positive nuclei in both the inner and outer nuclear layer cells per $1000 \mu \mathrm{m}$ was calculated for each group.

\section{Statistical Analysis}

Statistical analyses were performed using SPSS statistics, version 25 (INM Armonk, New York). Since the data was an abnormal distribution, the immunohistochemical staining intensity and the percentage of TUNEL-positive nuclei to the total ganglion cells per $100 \mu \mathrm{m}$ in the GCL were analyzed between each

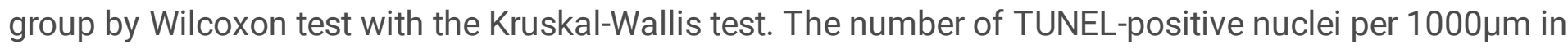


the inner nuclei layer (INL) and the outer nuclear layer (ONL) were analyzed by the one-way ANOVA test. The statistical significance was set at $P \otimes 0.05$.

\section{Results}

\section{Expression of VEGF in the neural retina}

VEGF is an important angiogenic factor and is also a downstream factor of HIF-1a. We explored the expression characteristics of VEGF in each group. The VEGF protein was almost undetectable in the 0 hour control group (Fig. 2A) and the 14-hour hypoxia alone group (Fig. 2B). The strong immunohistochemical staining of VEGF in the NFL/GCL was found in the vehicle treatment group (Fig. 2C). Compared to the vehicle treatment group, a little weaker immunohistochemical staining of VEGF in the NFL/GCL was found in both the PEDF treatment group (Fig. 2D) and the Avastin treatment group (Fig. 2E). The weak immunohistochemistry staining of VEGF was shown in the PEDF/Avastin treatment group (Fig. 2F). The statistical results of immunohistochemical staining intensity of VEGF in the neural retina (Fig. 2G). The staining intensity was significantly reduced in the PEDF/Avastin treatment group $(0,1)$ compared to the vehicle treatment group $(1,3)(P \otimes 0.001)$ and the PEDF treatment group $(1,3)$ $(P \otimes 0.001)$. The staining intensity was significantly reduced in the Avastin treatment group $(0,2)$ than the vehicle treatment group $(P=0.01)$.

\section{Expression of VEGF in the RPE layer and the choroidal vessels}

The immunohistochemical staining of VEGF was weak in the RPE cells and the choroidal vessels in the 0hour control group (Fig. 3A) and the 14-hour hypoxia alone group (Fig. 3B). The immunohistochemical staining of VEGF was increased in the vehicle treatment group (Fig. 3C) and the PEDF treatment group (Fig. 3D). Weak immunohistochemical staining of VEGF was found in the choroidal vessel walls of the Avastin treatment group (Fig. 3E). The VEGF in the RPE cells and the choroidal vessels was nearly negative in the PEDF/Avastin treatment group (Fig. 3F). The combined therapeutic approach significantly reduced VEGF in the RPE and the choroidal vessels. Thus, there might be a synergistic effect between PEDF and Avastin in inhibiting VEGF expression. The statistical results of immunohistochemical staining intensity of VEGF in the RPE layer (Fig. 4G). The staining intensity was significantly reduced in the PEDF/Avastin treatment group $(0,1)$ compared to the PEDF treatment group $(0,1)(P=0.004)$, the vehicle treatment group $(0,1)(P=0.004)$, and the control group $(0,1)(P=0.016)$. The statistical results of immunohistochemical staining intensity of VEGF in the choroid (Fig. $4 \mathrm{H}$ ). The staining intensity was significantly reduced in the PEDF/Avastin treatment group $(0,0)$ compared to the vehicle treatment group $(0,1)(P \otimes 0.001)$ and the 0 -hour control group $(0,1)(P=0.014)$. The staining intensity was significantly reduced in the PEDF treatment group $(0,1)$ compared to the vehicle treatment group $(P=0.003)$. The value is described by percentile (M1, M3).

\section{Immunoreactivity of GFAP in the neural retina}


GFAP is widely used as a molecular indicator for retinal stress. In the 0 -hour control group, there was almost no GFAP-immunopositive staining in the neural retina (Fig. 4A). After 14 hours of hypoxia, the weak immunohistochemical staining of GFAP was detected in the NFL of the 14-hour hypoxia alone group (Fig. 4B). The strongest immunohistochemical staining of GFAP was found in the NFL/GCL of the vehicle treatment group (Fig. 4C). Compared to the vehicle treatment group, both the PEDF treatment group (Fig. 4D) and the Avastin treatment group (Fig. 4E) showed a little less immunohistochemical staining of GFAP in the NFL/GCL. The weak GFAP-immunopositive staining was observed in the NFL of the PEDF/Avastin treatment group (Fig. 4F). Immunohistochemical staining intensity of GFAP in the neural retina (Fig. 4G-I). Compared to the 0 -hour control group $(1,3)$ and the 14 -hour ischemia alone group $(1,2)$, the staining intensity was significantly increased in the vehicle treatment group $(3,3)$ $(P \otimes 0.001)$, the PEDF treatment group $(3,3)(P \otimes 0.001)$ and the Avastin treatment group $(2.5,3)(P \otimes 0.001)$ (Fig. $5 \mathrm{G}$ and $\mathrm{H}$ ). The staining intensity was significantly reduced in the PEDF/Avastin treatment group (1, 3) compared to the vehicle treatment group, the PEDF treatment group and the Avastin treatment group (Pख0.001) (Fig. 5I). The value was described by percentile (M1, M3).

\section{The binding position of human PEDF in the retina}

Immunohistochemical staining of human PEDF was done in the PEDF treatment group and the PEDF/Avastin treatment group to detect the binding position in the retina. PEDF mainly combined to the $\mathrm{NFL} / \mathrm{GCL}$, the inner nuclei layer (INL) and the rod and cones layer (RCL) of the retina (Fig. 5A and B). These binding positions may help PEDF to exert its neuroprotective effects in the neural retina.

\section{Expression of PEDF in the RPE cells and the choroidal vessels}

Immunohistochemical staining of PEDF in the RPE layer and the choroid (Fig. 6A-F). The PEDF staining was detected in the RPE layer and it was nearly negative in the choroid of the 0 -hour control (Fig. 6A), the 14-hour ischemia alone group (Fig. 6B) and the Avastin treatment group (Fig. 6E). The immunohistochemical staining of PEDF was nearly negative in the RPE and the choroid of the vehicle treatment group (Fig. 6C). The immunohistochemical staining in the RPE layer and the choroidal vessel was found in the PEDF treatment group (Fig. 6D) and the PEDF/Avastin treatment group (Fig. 6F). Immunohistochemical staining intensity of PEDF in the RPE layer (Fig. 6G) and the choroid (Fig. 6H) for each group. Compared to the 0 -hour control group $(1,2)$, the 14 -hour ischemia alone group $(0,2)$, the vehicle treatment group $(0,1)$ and the Avastin treatment group $(0,1)$, the staining intensity of PEDF in the RPE cells was significantly increased in the PEDF treatment group $(2,3)(P \otimes 0.001)$ and the PEDF/Avastin treatment group $(1,3)(P \otimes 0.001)$ (Fig. 6G). Comparing to the 0 -hour control group $(0,0)$, the 14 -hour ischemia alone group $(0,0)$, the vehicle treatment group $(0,0)$ and the Avastin treatment group $(0,0)$, the staining intensity of PEDF in the choroid was significantly increased in the PEDF treatment group $(0,1)$ $(P \otimes 0.001)$ and the PEDF/Avastin treatment group $(0,1)(P \otimes 0.001)($ Fig. $6 \mathrm{H})$. The value was described by percentile (M1, M3). 


\section{TUNEL-positive nuclei were reduced in the PEDF \& Avastin treatment group}

To determine ganglion cell loss by programmed cell death, we carried out terminal deoxynucleotidyl transferase dUTP nick end labeling (TUNEL) assay. TUNEL-positive nuclei were mainly located in the inner retina, especially the GCL (Fig. 7G-L). However, TUNEL-positive nuclei were rarely observed in the outer nuclear layer (Fig. 7M-R). In the GCL, the percentage of TUNEL-positive nuclei per $100 \mu \mathrm{m}$ was significantly reduced in the PEDF/Avastin treatment group $(2.21,2.55)$ compared to the 14-hour ischemia alone group $(2.90,3.69)(P=0.001)$, the vehicle treatment group $(3.16,3.77)(P \otimes 0.001)$ and the Avastin treatment group $(2.89,3.69)(P=0.002)$ (Fig. 9A). The percentage of TUNEL-positive nuclei per $100 \mu \mathrm{m}$ was significantly reduced in the PEDF treatment group $(2.35,2.89)$ compared to the vehicle treatment group $(3.16,3.77)(P=0.004)$. The number of TUNEL-positive nuclei in the inner nuclear layer cells per $1000 \mu \mathrm{m}$ (Fig. 7B). There is a significant difference between the 14-hour ischemia alone group (38.96 \pm $9.00)$ and the PEDF/Avastin treatment group $(24.30 \pm 6.21)(P=0.009)$. The number of TUNEL-positive nuclei was significantly reduced in the PEDF treatment group $(16.99 \pm 6.60)$ compared to the 14 -hour ischemia alone group $(P \otimes 0.001)$, the vehicle treatment group $(32.69 \pm 10.38)(P=0.005)$ and the Avastin treatment group $(32.01 \pm 9.42)(P=0.006)$. The number of TUNEL-positive nuclei per $1000 \mu \mathrm{m}$ in the outer nuclear layer (ONL) (Fig. 9C). Very few apoptotic cells were found in the ONL for each group. There was a significant difference between the control group $(0.98 \pm 1.23)$ and the 14-hour ischemia alone group (2.86 $\pm 1.46)(P=0.003)$, the vehicle treatment group $(2.57 \pm 1.24)(P=0.01)$. The value is described by percentile $(M 1, M 3)$ and (Mean $\pm S D)$.

\section{Discussion}

This study shows that PEDF combined with anti-VEGF therapy can reduce Müller glial cell and astrocyte activation and neuro-protect RGCs ex vivo in rat eyes under ischemia. PEDF/Anti-VEGF therapy prevented the expression of VEGF in the neural retina and the choroidal vessels. PEDF and Anti-VEGF have a synergistic effect in inhibiting VEGF. Synergistic inhibition of VEGF may be more effective on ischemiainduced retinal neovascularization. The results provide insights into a new potential treatment method for the ischemia-related retinal diseases.

VEGF is well known as an important angiogenesis factor and is related to retinal ischemia and intraocular angiogenesis [19]. The pathological activation of the hypoxia inducible factor (HIF) pathway leads to expression of VEGF and causes ocular neovascularization [20]. In our experiment, the immunostaining of VEGF was nearly negative in the neural retina of 14 hours hypoxia non-treated eyes. The vehicle-treated eyes showed the strongest immunostaining of VEGF in the retinal nerve fiber layer (NFL)/GCL, while the PEDF/Avastin-treated therapy significantly reduced the expression of VEGF in the neural retina compared to the vehicle-treated and the PEDF-treated eyes. In addition, the staining intensity of VEGF in the neural retina of the PEDF/Avastin-treated eyes was very similar to the 0 -hour control eyes. These indicate that the combination of PEDF and Avastin has a synergistic effect in attenuating the expression of VEGF in 
the neural retina. Thus, the combination therapy may be a promising method to treat retinal neovascularization induced by ischemia/hypoxia. In addition, the distribution of VEGF in the neural retina is restricted to the retinal nerve fiber layer (NFL) and the retinal ganglion cell layer (GCL). As previously reported, this is the location of astrocytes and the end-feet of Müller cells [21]. VEGF plays a vital role in ischemia-induced retinal neovascularization, vascular leakage and inflammation [22]. So, blocking VEGF signaling pathway in Müller cells may be a method to treat retinal neovascular diseases.

RPE cells are the major source of VEGF in the eye and it plays an important role in maintaining the homeostasis of the retina and the choroid [23]. In response to hypoxia, RPE cells release various growth factors such as VEGF and result in angiogenesis [24]. In our experiment, the VEGF was weakly detected in the RPE layer and the choroidal vessels in the 0-hour control eyes, as a physiological amount of VEGF is required for the homeostasis in the retina and the choroid [25]. VEGF was increased in the RPE layer and the choroidal vessels in the vehicle-treated eyes. However, the PEDF/Avastin therapy significantly attenuated the expression of VEGF in both the RPE cell layer and the choroidal vessels. Ischemia induced expression of VEGF in the RPE and the choroidal vessels are important factors for the formation of CNV or AMD $[26,27]$. So, the PEDF/Avastin treatment may be a potential therapeutic method for the CNV or wet age-related macular degeneration (AMD).

GFAP is used as a marker of injury in the retina and indicates astrocyte or Müller cell activation [28]. In this study, the immunohistochemical staining of GFAP was weakly detected in the 0-hour control retina. surprisingly and unexpectedly, even weaker GFAP immunopositive staining was detected in the retinal nerve fiber layer (NFL)/GCL of the 14 hours hypoxia non-treated eyes. The possible explanation might be that the activation of astrocytes and Müller cells was diminished under hypothermia. A previous study also reported similar results that hypothermia $\left(20^{\circ} \mathrm{C}\right)$ significantly reduced the amount of GFAP in the retina [29]. The reason may be that hypothermia attenuates gliosis and partially overlays ischemiainduced glial activation. The intensity of GFAP immunoreactivity was significantly increased in the intravitreal treatment eyes, except for the PEDF/Avastin treatment eyes. The PEDF/Avastin treatment eyes showed very weak GFAP staining in the NFL/GCL, indicating that PEDF combined with an anti-VEGF drug (Avastin) significantly decreased and avoided ischemia-induced retinal Müller cell and astrocytes damage. Müller cell can express PEDF, which has a neuroprotective effect on retinal neural cells. Protecting Müller cell from damage may be one of the mechanisms by which intravitreal PEDF protein alone or PEDF/anti-VEGF therapy protect ganglion cells. These results provide a new insight into the development of a potential therapeutic approach to treat ischemic retinopathy.

Interestingly, the immune-positive staining of VEGF and GFAP are all located in the NFL and GCL of the retina for the four intravitreal injection groups, and the positive position represents astrocytes and Müller glia end-feet. It was unfortunate that we did not do the co-immunohistochemistry staining, but at least we found the consistent feature of this staining position. VEGF expression was found in the nerve fiber layer (NFL) in hypoxia-induced retinal injury, and the double immunofluorescence labeling showed that GFAP and VEGF were complete colocalization [30]. It is not clear which factor increased at the earliest stages under ischemia, but the combination therapy (PEDF/Avastin) reduced their expression in NFL and GCL. 
Immunohistochemical staining of PEDF was performed in both the PEDF-treated and the PEDF/Avastintreated groups to examine its location and function in the retina and the choroid. It is noteworthy that the intravitreally injected PEDF protein was detected in the GCL, INL, rod and cones layer, RPE layer and sometimes in the choroidal vessel walls. The results showed that the intravitreally injected PEDF protein could bind in the retinal GCL, the RPE cells and the choroidal vessel and this helped PEDF in protecting ganglion cells and avoiding choroidal neovascularization under ischemia.

This study investigated the effects of different intravitreal injections on the survival of retinal ganglion cells (RGCs). Compared to the vehicle-treated group, both PEDF-treated and PEDF/Avastin-treated groups showed a significantly decreased number of TUNEL-positive nuclei, and the double treatment group had the least TUNEL-positive nuclei. PEDF protects the RGCs from cell death ex vivo. This may be partly attributed to the neuroprotective effect of exogenous PEDF [31], and the combination of PEDF and Avastin has a synergistic effect. Alternatively, the double treatment downregulates GFAP expression in the ganglion cell layer and this means that the combined therapeutic approach prevents the degenerative Müller glial response. We speculate that the neuroprotective effect on ganglion cells may be achieved by maintaining the normal physiological functions of Müller cells. Müller cell derived neurotrophic and neuroprotective mediators such as PEDF can support neuronal survival [32]. In addition, PEDF itself has been shown to prevent the degenerative glial response in the retina by reducing the inflammatory state of Müller glial cells [33]. Retinal glutamate is degraded in Müller cells by glutamine synthetase (GS) through synthesis of glutamine [34], and the dysfunction of Müller cells may lead to excessive accumulation of glutamate and glutamate toxicity in the retina. It is interesting that the PEDF/Avastin treatment has a protective effect on retinal Müller cells and this may help maintain the normal glutamate metabolism. Enhancing the function of Müller cells may be useful to promote RGC survival under ischemic retinopathy.

In conclusion, PEDF combined with an anti-VEGF drug (Avastin) significantly reduces VEGF in the retina and choroid of the ischemic ex vivo eye model. This helps to improve the adverse effects of hypoxia on the retina and choroid and prevent the formation of new blood vessels. GFAP in the retinal nerve fiber layer and GCL was significantly reduced after the combined therapeutic approach, indicating the protective effect of PEDF/Anti-VEGF on Müller glial cells. The normal physiological functions of Müller cells may play an important role in protecting retinal ganglion cells, and the combined therapeutic approach may protect ganglion cell survival under retinal ischemia by protecting Müller cells. Thus, PEDF combined with anti-VEGF therapy is a potential method to treat retinal and choroidal ischemic diseases.

\section{Conclusions}

The combination of PEDF and an anti-VEGF drug (Avastin) effectively reduces the level of VEGF and GFAP in the neural retina, the RPE cell layer and the choroidal vessels and it is a possible therapeutic strategy against ischemic retinal and choroidal diseases. PEDF alone or PEDF/Anti-VEGF shows antiapoptosis function for the retinal neural cells. Thus, PEDF combines with an anti-VEGF therapy may be a favorable method to treat retinal and choroidal ischemic diseases compared to anti-VEGF alone. 


\section{Abbreviations}

PEDF: pigment epithelium derived factor; VEGF: vascular endothelial growth factor; CNV: choroidal neovascularization; DMEM: Dulbecco's modified eagle medium; GFAP: glial fibrillary acidic protein; NFL: nerve fiber layer; GCL: ganglion cell layer; BRVO: branch retinal vein occlusion; NV: neovascularization; RGCs: retinal ganglion cells; HIF: hypoxia inducible factor; AMD: age-related macular degeneration; GS: glutamine synthetase.

\section{Declarations}

\section{Acknowledgments}

The paper is funded by the University of Tübingen, Germany.

\section{Author's Contributions}

$\mathrm{XL}$ designed and executed the study, performed experiments, analyzed the data, wrote the manuscript, and approved the final manuscript.

\section{Availability of data and materials}

The data used to support the findings of this study are available from the corresponding author upon request.

\section{Ethics approval and consent to participate}

All experiments were performed according to standard protocols, in compliance with the Guide of the protection agency and supervision of veterinarians of the University of Tübingen.

\section{Consent for publication}

Not applicable.

\section{Conflicts of interest}

The author declares that there are no competing interests.

\section{Funding}

Not applicable

\section{References}

1. Noueihed B, Rivera JC, Dabouz R, Abram P, Omri S, Lahaie I, et al. Mesenchymal Stromal Cells Promote Retinal Vascular Repair by Modulating Sema3E and IL-17A in a Model of Ischemic Retinopathy. Front Cell 
Dev Biol. 2021;9:630645.

2. Falavarjani KG, Nguyen QD. Adverse events and complications associated with intravitreal injection of anti-VEGF agents: a review of literature. Eye (Lond). 2013;27:787-94.

3. Wang J, Wang X, Gao Y, Lin Z, Chen J, Gigantelli J, et al. Stress Signal Regulation by Na/K-ATPase As a New Approach to Promote Physiological Revascularization in a Mouse Model of Ischemic Retinopathy. Invest Ophthalmol Vis Sci. 2020;61:9.

4. Tokunaga CC, Mitton KP, Dailey W, Massoll C, Roumayah K, Guzman E, et al. Effects of anti-VEGF treatment on the recovery of the developing retina following oxygen-induced retinopathy. Invest Ophthalmol Vis Sci. 2014;55:1884-92.

5. Stone J, Itin A, Alon T, Pe'er J, Gnessin H, Chan-Ling T, et al. Development of retinal vasculature is mediated by hypoxia-induced vascular endothelial growth factor (VEGF) expression by neuroglia. J Neurosci. 1995;15:4738-47.

6. Lutty GA, McLeod DS, Bhutto I, Wiegand SJ. Effect of VEGF trap on normal retinal vascular development and oxygen-induced retinopathy in the dog. Invest Ophthalmol Vis Sci. 2011;52:4039-47.

7. Shields CL, Shields JA, Cater J, Lois N, Edelstein C, Gündüz K, et al. Transpupillary thermotherapy for choroidal melanoma: tumor control and visual results in 100 consecutive cases. Ophthalmology. 1998;105:581-90.

8. Sternberg P Jr, Lewis H. Photodynamic therapy for age-related macular degeneration: a candid appraisal. Am J Ophthalmol. 2004;137:483-5.

9. Hayreh SS, Zimmerman MB, Kimura A, Sanon A. Central retinal artery occlusion. Retinal survival time. Exp Eye Res. 2004;78:723-36.

10. Tombran-Tink J, Johnson LV. Neuronal differentiation of retinoblastoma cells induced by medium conditioned by human RPE cells. Invest Ophthalmol Vis Sci. 1989;30:1700-7.

11. Dawson DW, Volpert OV, Gillis P, Crawford SE, Xu H, Benedict W, et al. Pigment epithelium-derived factor: a potent inhibitor of angiogenesis. Science. 1999;285:245-8.

12. Vigneswara V, Berry M, Logan A, Ahmed Z. Pigment epithelium-derived factor is retinal ganglion cell neuroprotective and axogenic after optic nerve crush injury. Invest Ophthalmol Vis Sci. 2013;54:2624-33.

13. Eichler W, Savković-Cvijić H, Bürger S, Beck M, Schmidt M, Wiedemann P, et al. Müller Cell-Derived PEDF Mediates Neuroprotection via STAT3 Activation. Cell Physiol Biochem. 2017;44:1411-1424.

14. Comitato A, Subramanian P, Turchiano G, Montanari M, Becerra SP, Marigo V. Pigment epitheliumderived factor hinders photoreceptor cell death by reducing intracellular calcium in the degenerating 
retina. Cell Death Dis. 2018;9:560.

15. Yang J, Duh EJ, Caldwell RB, Behzadian MA. Antipermeability function of PEDF involves blockade of the MAP kinase/GSK/beta-catenin signaling pathway and uPAR expression. Invest Ophthalmol Vis Sci. 2010;51:3273-80.

16. Zhang SX, Wang JJ, Gao G, Parke K, Ma JX. Pigment epithelium-derived factor downregulates vascular endothelial growth factor (VEGF) expression and inhibits VEGF-VEGF receptor 2 binding in diabetic retinopathy. J Mol Endocrinol. 2006;37:1-12.

17. Pe'er J, Shweiki D, Itin A, Hemo I, Gnessin H, Keshet E. Hypoxia-induced expression of vascular endothelial growth factor by retinal cells is a common factor in neovascularizing ocular diseases. Lab Invest. 1995;72:638-45.

18. Gao G, Li Y, Zhang D, Gee S, Crosson C, Ma J. Unbalanced expression of VEGF and PEDF in ischemiainduced retinal neovascularization. FEBS Lett. 2001;489:270-6.

19. DeNiro M, Alsmadi O, Al-Mohanna F. Modulating the hypoxia-inducible factor signaling pathway as a therapeutic modality to regulate retinal angiogenesis. Exp Eye Res. 2009;89:700-17.

20. Vavilala DT, Ponnaluri VK, Kanjilal D, Mukherji M. Evaluation of anti-HIF and anti-angiogenic properties of honokiol for the treatment of ocular neovascular diseases. PLoS One. 2014;9:e113717.

21. Håkansson G, Gesslein B, Gustafsson L, Englund-Johansson U, Malmsjö M. Hypoxia-inducible factor and vascular endothelial growth factor in the neuroretina and retinal blood vessels after retinal ischemia. J Ocul Biol Dis Infor. 2010;3:20-9.

22. Lin M, Chen Y, Jin J, Hu Y, Zhou KK, Zhu M, et al. Ischaemia-induced retinal neovascularisation and diabetic retinopathy in mice with conditional knockout of hypoxia-inducible factor-1 in retinal Müller cells. Diabetologia. 2011;54:1554-66.

23. Puddu A, Sanguineti R, Traverso CE, Viviani GL, Nicolò M. Response to anti-VEGF-A treatment of retinal pigment epithelial cells in vitro. Eur J Ophthalmol. 2016;26:425-30.

24. Vadlapatla RK, Vadlapudi AD, Pal D, Mukherji M, Mitra AK. Ritonavir inhibits HIF-1a-mediated VEGF expression in retinal pigment epithelial cells in vitro. Eye (Lond). 2014;28:93-101.

25. Miwa Y, Hoshino Y, Shoda C, Jiang X, Tsubota K, Kurihara T. Pharmacological HIF inhibition prevents retinal neovascularization with improved visual function in a murine oxygen-induced retinopathy model. Neurochem Int. 2019;128:21-31.

26. Lin M, Hu Y, Chen Y, Zhou KK, Jin J, Zhu M, et al. Impacts of hypoxia-inducible factor-1 knockout in the retinal pigment epithelium on choroidal neovascularization. Invest Ophthalmol Vis Sci. 2012 Sep 14;53(10):6197-206. 
27. Sheridan CM, Pate S, Hiscott P, Wong D, Pattwell DM, Kent D. Expression of hypoxia-inducible factor1alpha and -2alpha in human choroidal neovascular membranes. Graefes Arch Clin Exp Ophthalmol. 2009;247:1361-7.

28. Kim CR, Kim JH, Park HL, Park CK. Ischemia Reperfusion Injury Triggers TNFa Induced-Necroptosis in Rat Retina. Curr Eye Res. 2017;42:771-779.

29. Klemm P, Hurst J, Dias Blak M, Herrmann T, Melchinger M, Bartz-Schmidt KU, et al. Hypothermia protects retinal ganglion cells against hypoxia-induced cell death in a retina organ culture model. Clin Exp Ophthalmol. 2019;47:1043-1054.

30. Kaur C, Sivakumar V, Foulds WS. Early response of neurons and glial cells to hypoxia in the retina. Invest Ophthalmol Vis Sci. 2006;47:1126-41.

31. Ogata N, Wang L, Jo N, Tombran-Tink J, Takahashi K, Mrazek D, Matsumura M. Pigment epithelium derived factor as a neuroprotective agent against ischemic retinal injury. Curr Eye Res. 2001;22:245-52.

32. Lange J, Yafai Y, Reichenbach A, Wiedemann P, Eichler W. Regulation of pigment epithelium-derived factor production and release by retinal glial (Müller) cells under hypoxia. Invest Ophthalmol Vis Sci. 2008;49:5161-7.

33. Shen X, Zhong Y, Xie B, Cheng Y, Jiao Q. Pigment epithelium derived factor as an anti-inflammatory factor against decrease of glutamine synthetase expression in retinal Müller cells under high glucose conditions. Graefes Arch Clin Exp Ophthalmol. 2010;248:1127-36.

34. Wang Y, Lu Q, Gao S, Zhu Y, Gao Y, Xie B, et al. Pigment epithelium-derived factor regulates glutamine synthetase and I-glutamate/l-aspartate transporter in retinas with oxygen-induced retinopathy. Curr Eye Res. 2015;40:1232-44.

\section{Figures}




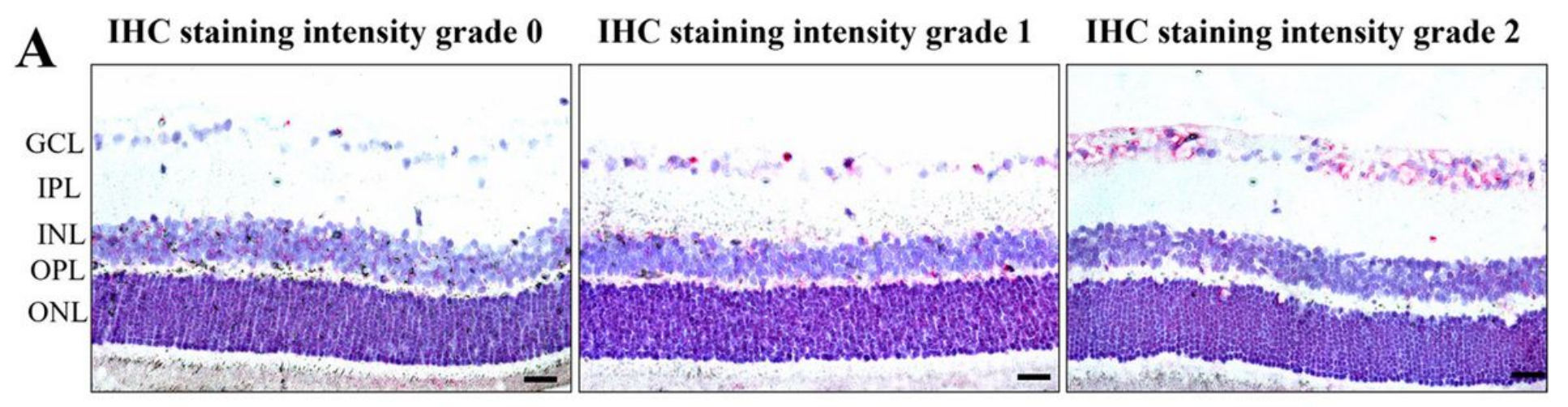

\section{IHC staining intensity grade 3 IHC staining intensity grade 4}
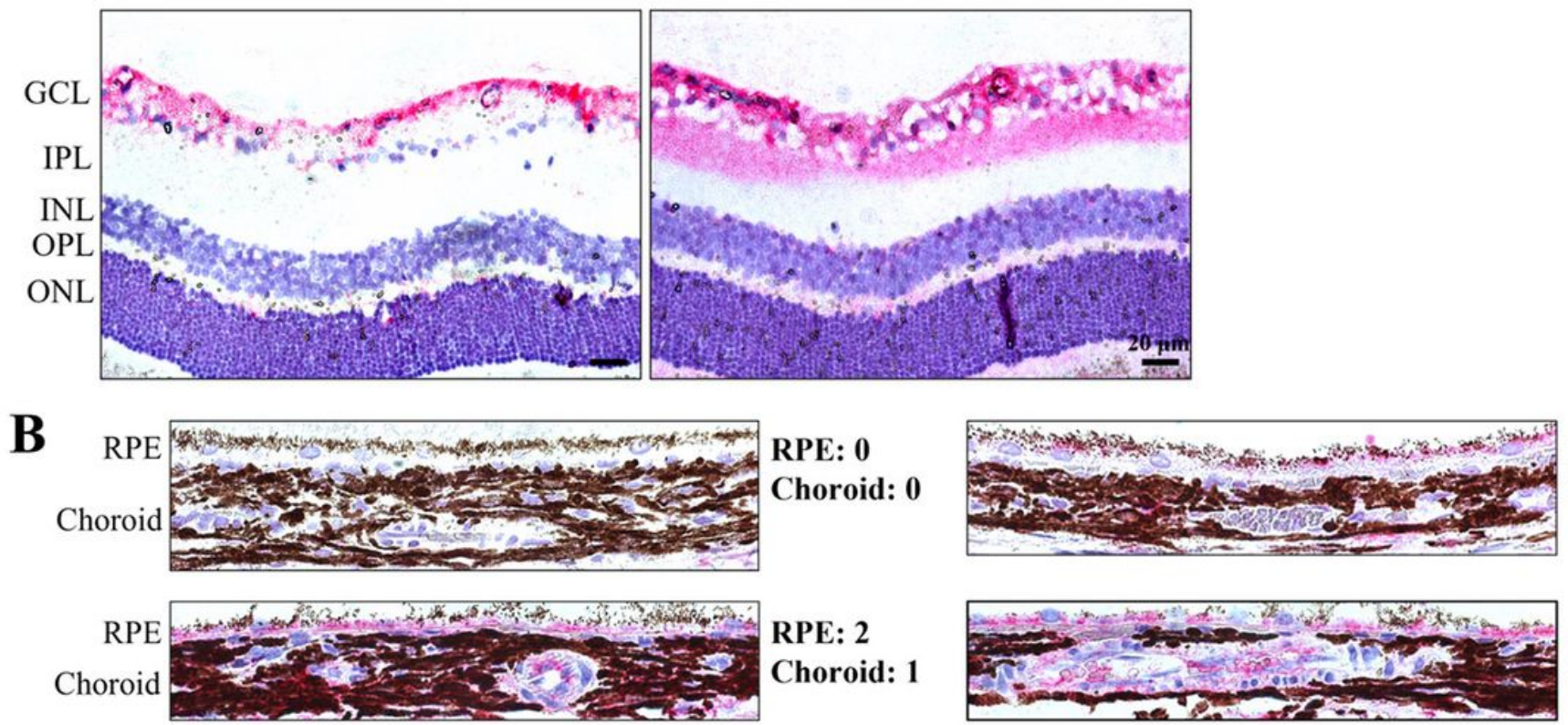

RPE: 1

Choroid: 0

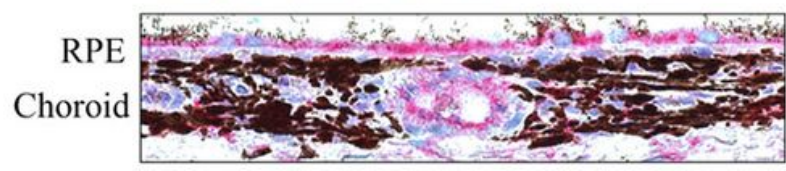

RPE: 3

Choroid: 3

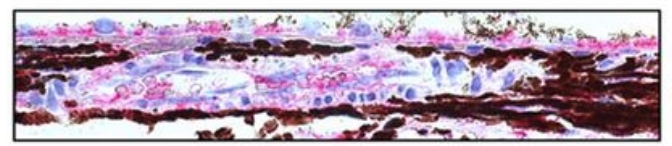

RPE: 2

Choroid: 2
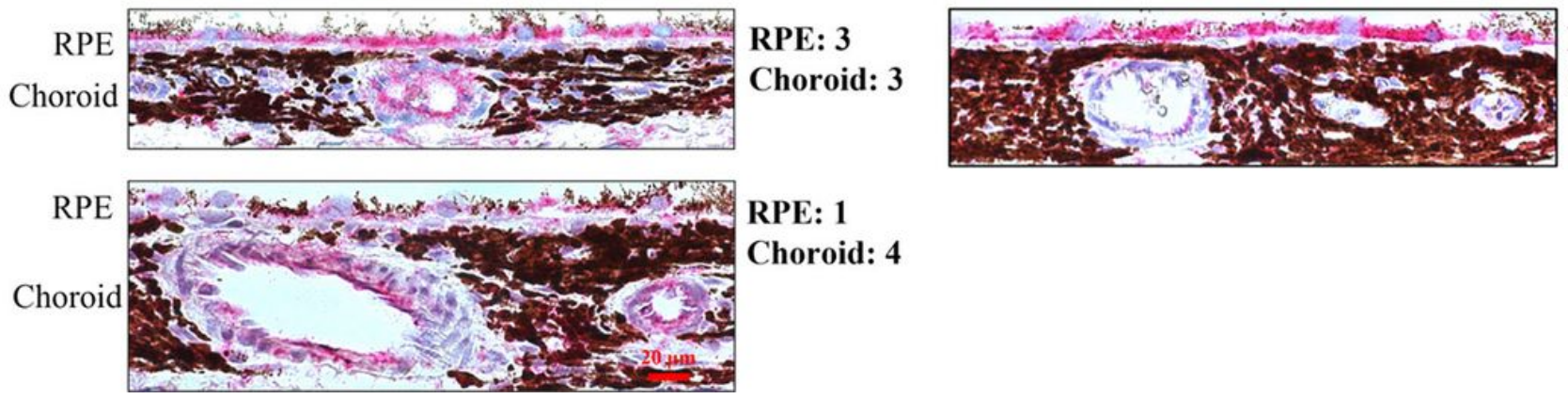

RPE: 4

Choroid: 1

\section{Figure 1}

Grading of the immunohistochemical staining intensity. Schematic diagram of the staining intensity in the neural retina (A), the RPE and choroidal vessel (B). The staining intensity gradually increases from grade 0 to grade 4 . Grade 0 is negative, without any staining. Grade 4 is positive, with the strongest degree of staining. IHC: immunohistochemical GCL: ganglion cell layer; IPL: inner plexiform layer; INL: inner nuclear layer; OPL: outer plexiform layer; ONL: outer nuclear layer; RPE: retinal pigmented epithelium. Scale bar $=20 \mu \mathrm{m}$. 


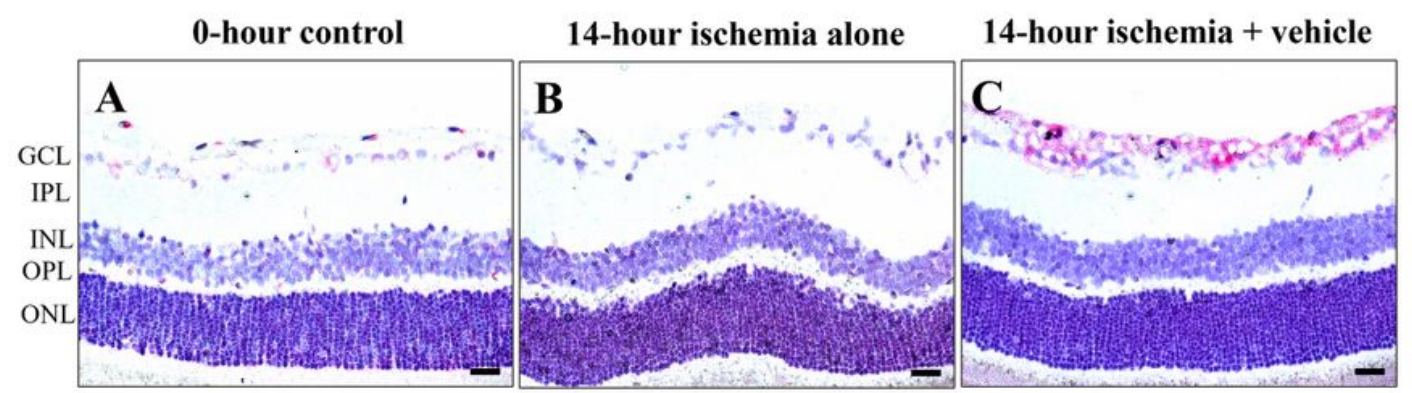

14-hour ischemia + $($ PEDF +

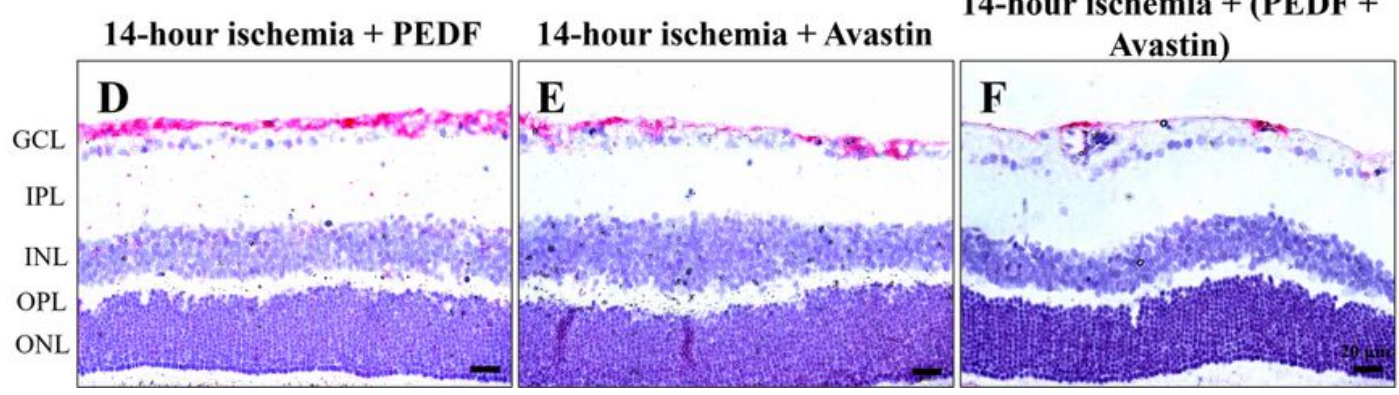

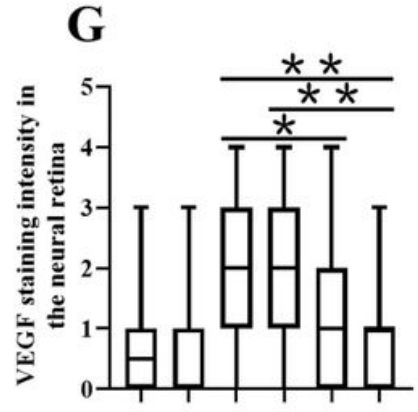

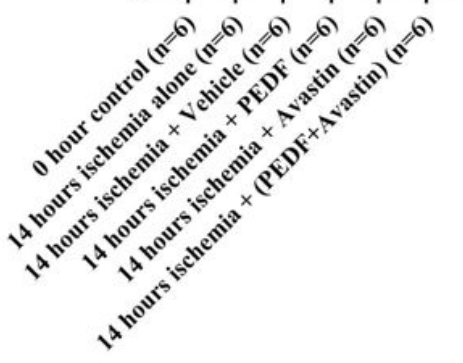

Figure 2

Representative images from immunohistochemical staining of VEGF in the posterior of the neural retina (A-F). The VEGF was nearly undetectable in the 0 -hour control group (A). Following 14 hours of hypoxia, there was hardly any positive staining of VEGF in the retina of 14 hours hypoxia alone group (B). The strongest immunohistochemical staining of VEGF in the GCL/NFL was found in the vehicle treatment group (C). VEGF-immunopositive staining was found in the GCL/NFL of the PEDF treatment group (D) and the Avastin treatment group (E). The weak immunohistochemistry staining of VEGF was shown in the PEDF/Avastin treatment group (F). Immunohistochemical staining intensity of VEGF in the neural

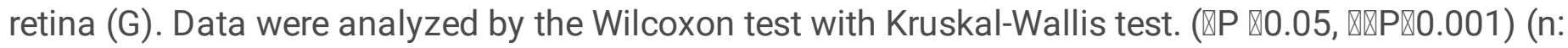
number of eyes). GCL: ganglion cell layer; IPL: inner plexiform layer; INL: inner nuclear layer; OPL: outer plexiform layer; ONL: outer nuclear layer; NFL: nerve fiber layer. Scale bar $=20 \mu \mathrm{m}$. 

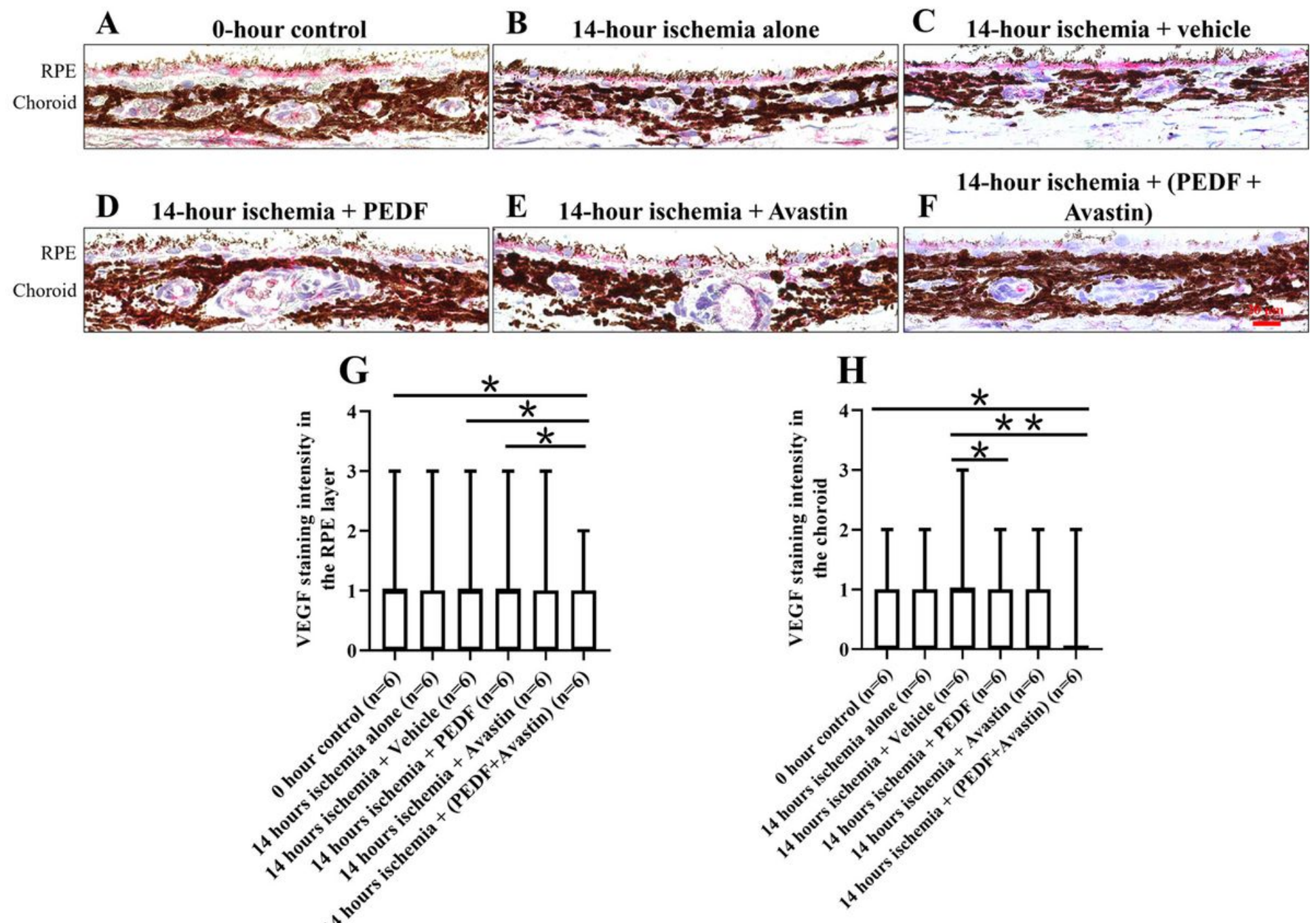

\section{Figure 3}

Immunohistochemical staining of VEGF in the RPE and choroid (A-F). The VEGF was weakly detected in the 0 -hour control group (A) and the 14 hours hypoxia alone group (B). The immunohistochemical staining of VEGF was found in the RPE and choroid vessel walls of the vehicle treatment group (C). The weak VEGF staining was found in the RPE cells and the choroid vessel walls of PEDF treatment group (D) and the Avastin treatment group (E). The immunohistochemical staining of VEGF in the choroid was nearly negative in the PEDF/Avastin treatment group (F). Immunohistochemical staining intensity of VEGF in the RPE layer $(G)$ and the choroidal vessel $(H)$. Data were analyzed by the Wilcoxon test with

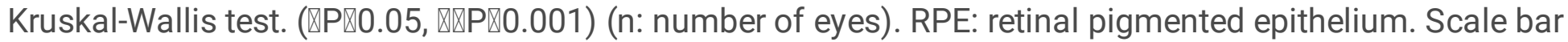
$=20 \mu \mathrm{m}$. 


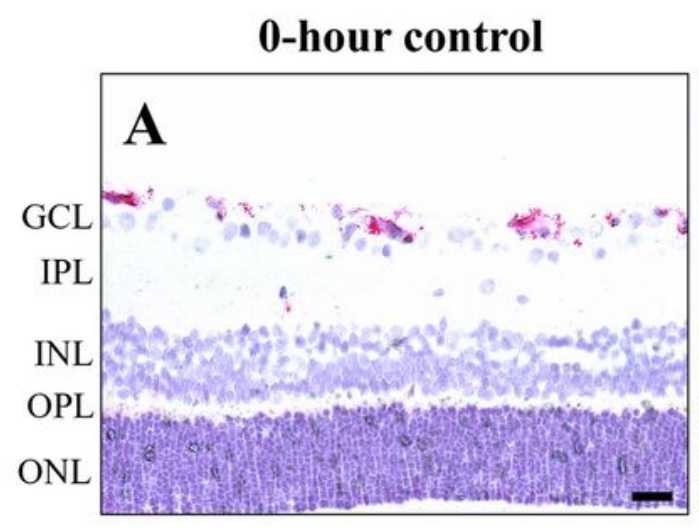

14-hour ischemia alone

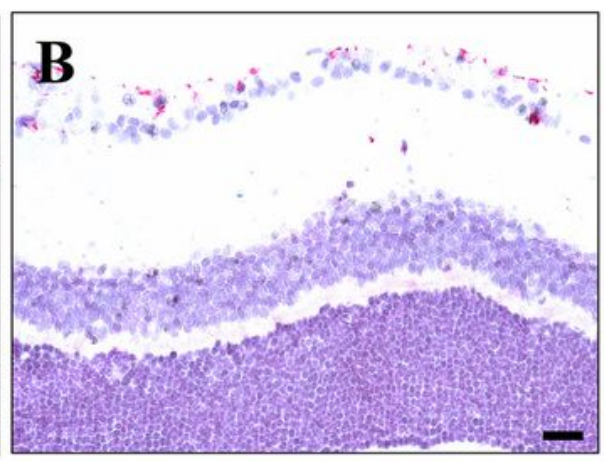

14-hour ischemia + PEDF

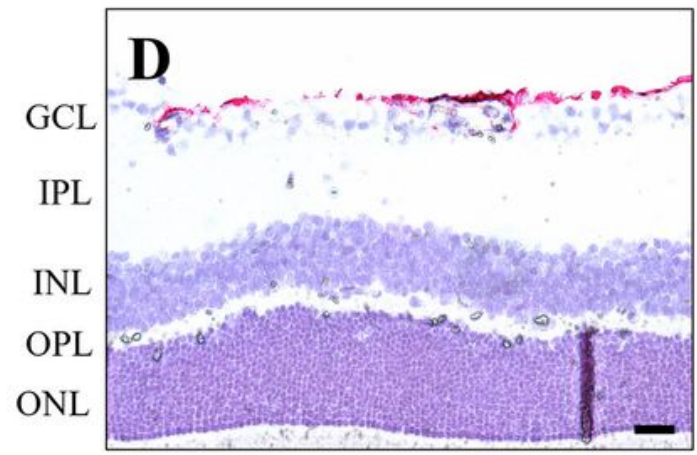

$\mathbf{G}$
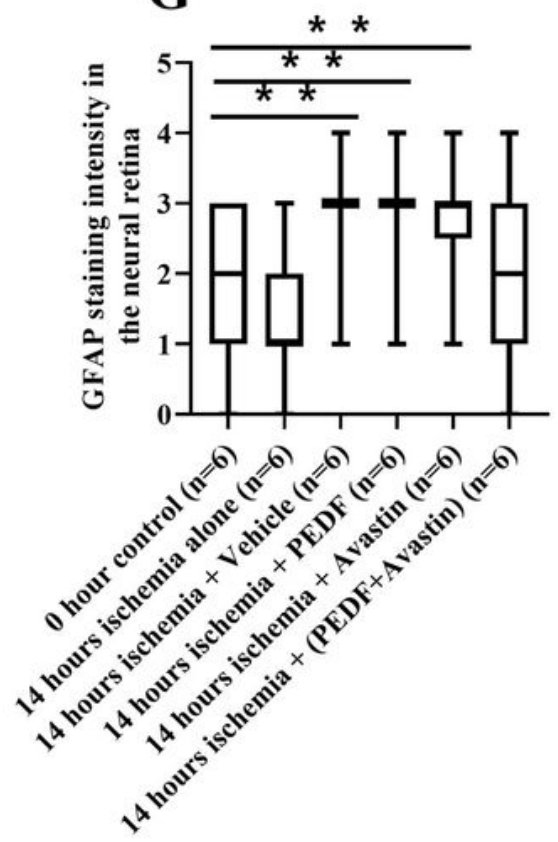

14-hour ischemia + Avastin

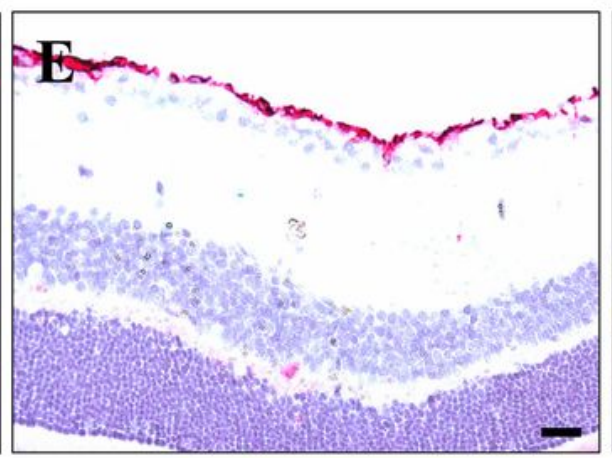

$\mathbf{H}$
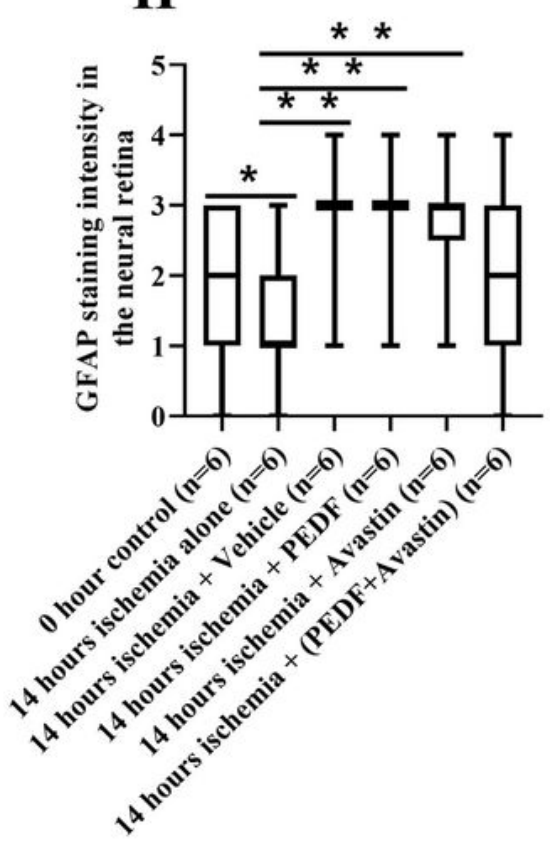

14-hour ischemia + vehicle

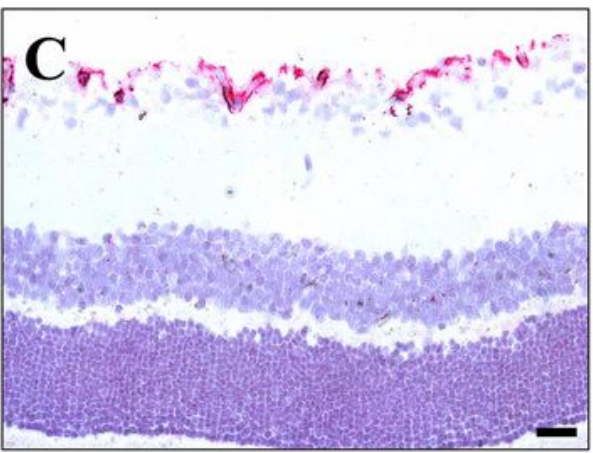

14-hour ischemia + (PEDF + Avastin)
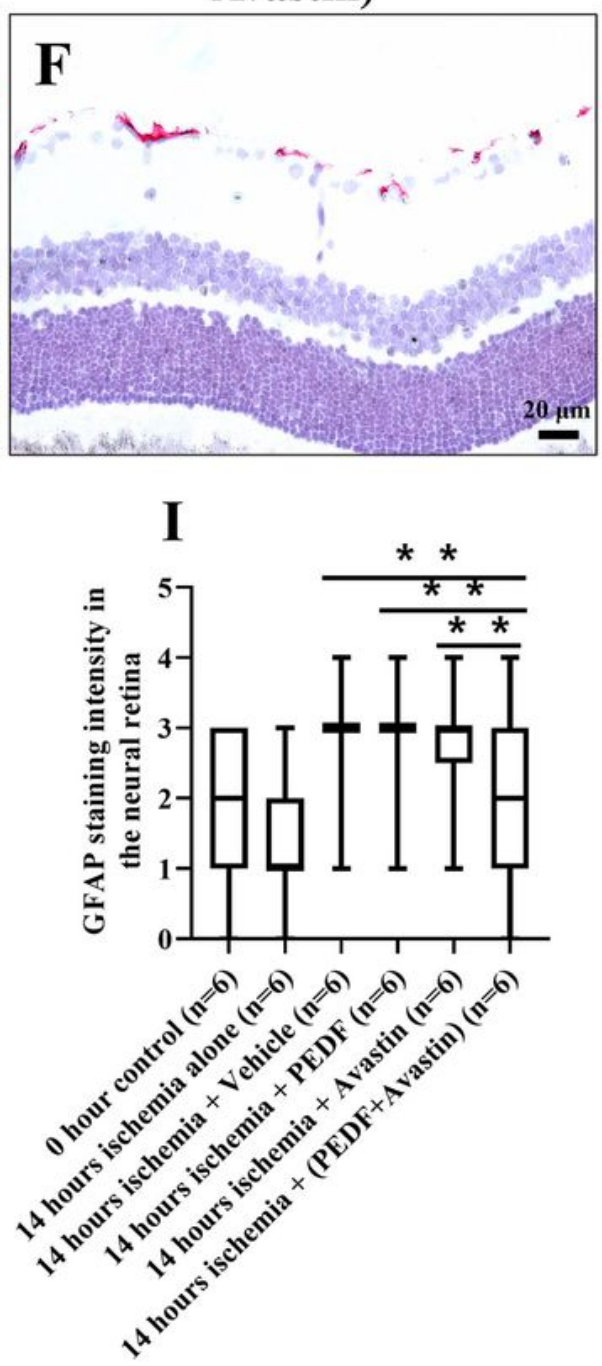

Figure 4

Representative images from immunohistochemical staining of GFAP in the retina (A-F). The GFAP was weakly detected in the 0 -hour control group $(A)$. Following 14 hours of hypoxia, weak GFAPimmunopositive staining was observed in the retinal nerve fiber layer (NFL)/GCL of the 14 hours hypoxia alone group (B). GFAP was massively upregulated in the NFL/GCL of the vehicle treatment group (C). The GFAP-immunopositive staining was found in the NFL/GCL of the PEDF treatment group (D) and the 
Avastin treatment group (E). The weak GFAP-immunopositive staining was detected in the NFL/GCL of the PEDF/Avastin treatment group (F). Immunohistochemical staining intensity of GFAP in the neural retina (G-I). Data were analyzed by the Wilcoxon test with Kruskal-Wallis test. (《षP®0.001) (n: number of eyes). GCL: ganglion cell layer; IPL: inner plexiform layer; INL: inner nuclear layer; OPL: outer plexiform layer; ONL: outer nuclear layer. Scale bar $=20 \mu \mathrm{m}$.

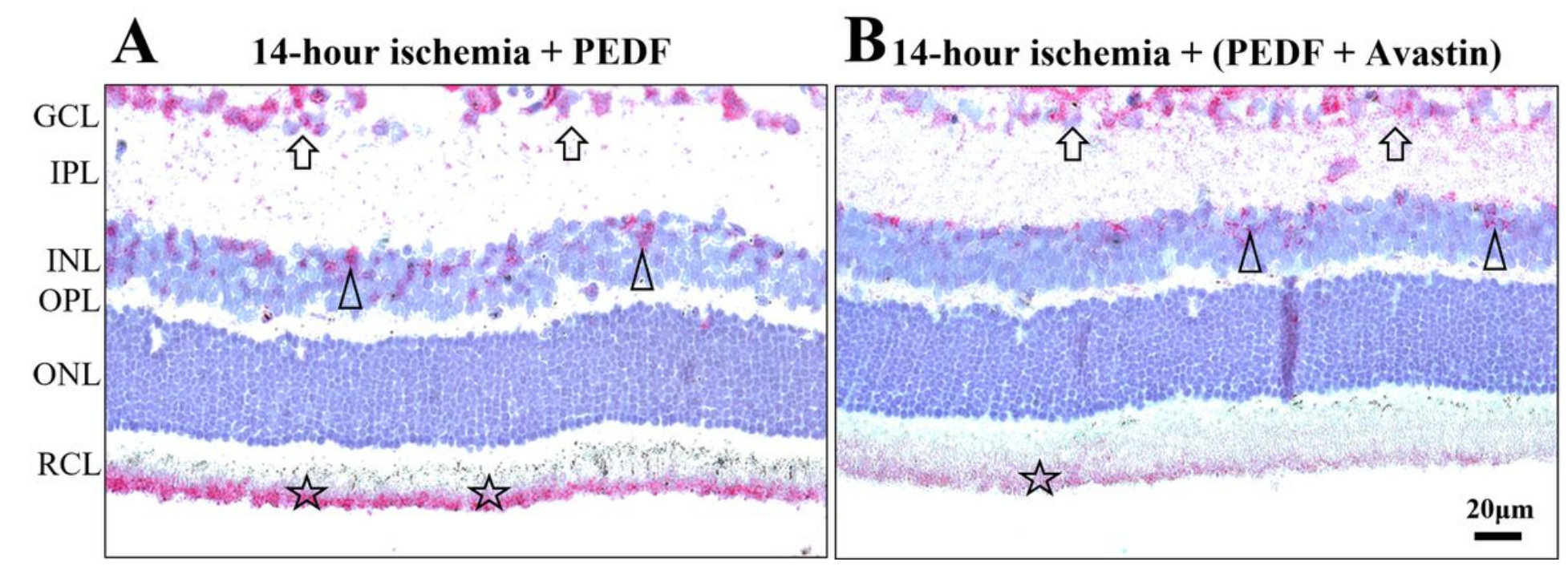

\section{Figure 5}

Immunohistochemical staining of PEDF in the retina. The PEDF was detected in the GCL (black arrow), the INL (black arrowhead) and the RCL (Star) of both the PEDF treatment group (A) and the PEDF/Avastin treatment group (B). GCL: ganglion cell layer; IPL: inner plexiform layer; INL: inner nuclear layer; OPL: outer plexiform layer; ONL: outer nuclear layer; RCL: rod and cones layer. Scale bar (retina) $=20 \mu \mathrm{m}$. 

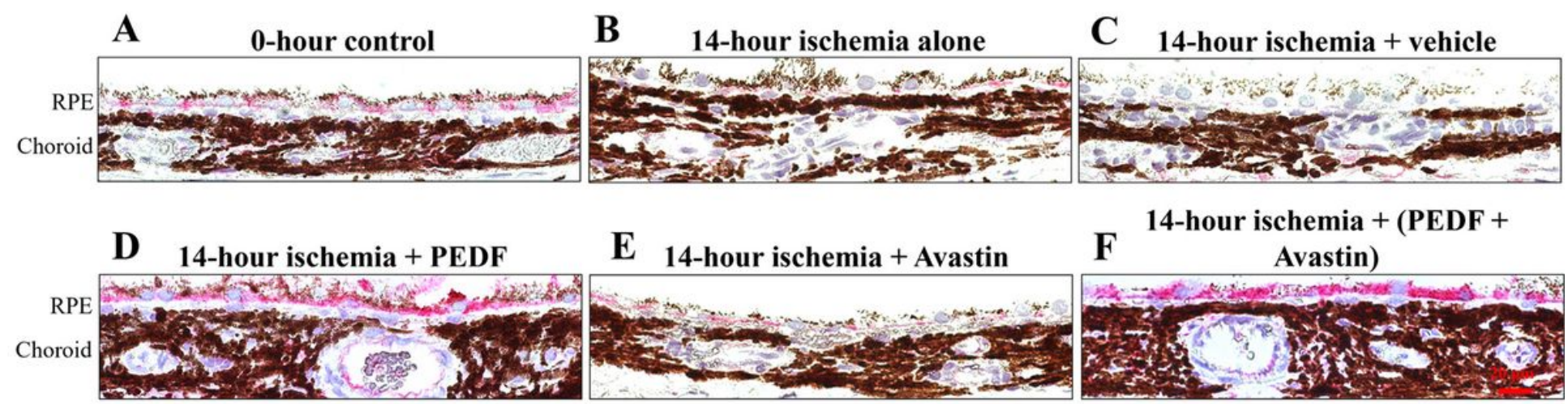

E 14-hour ischemia + Avastin
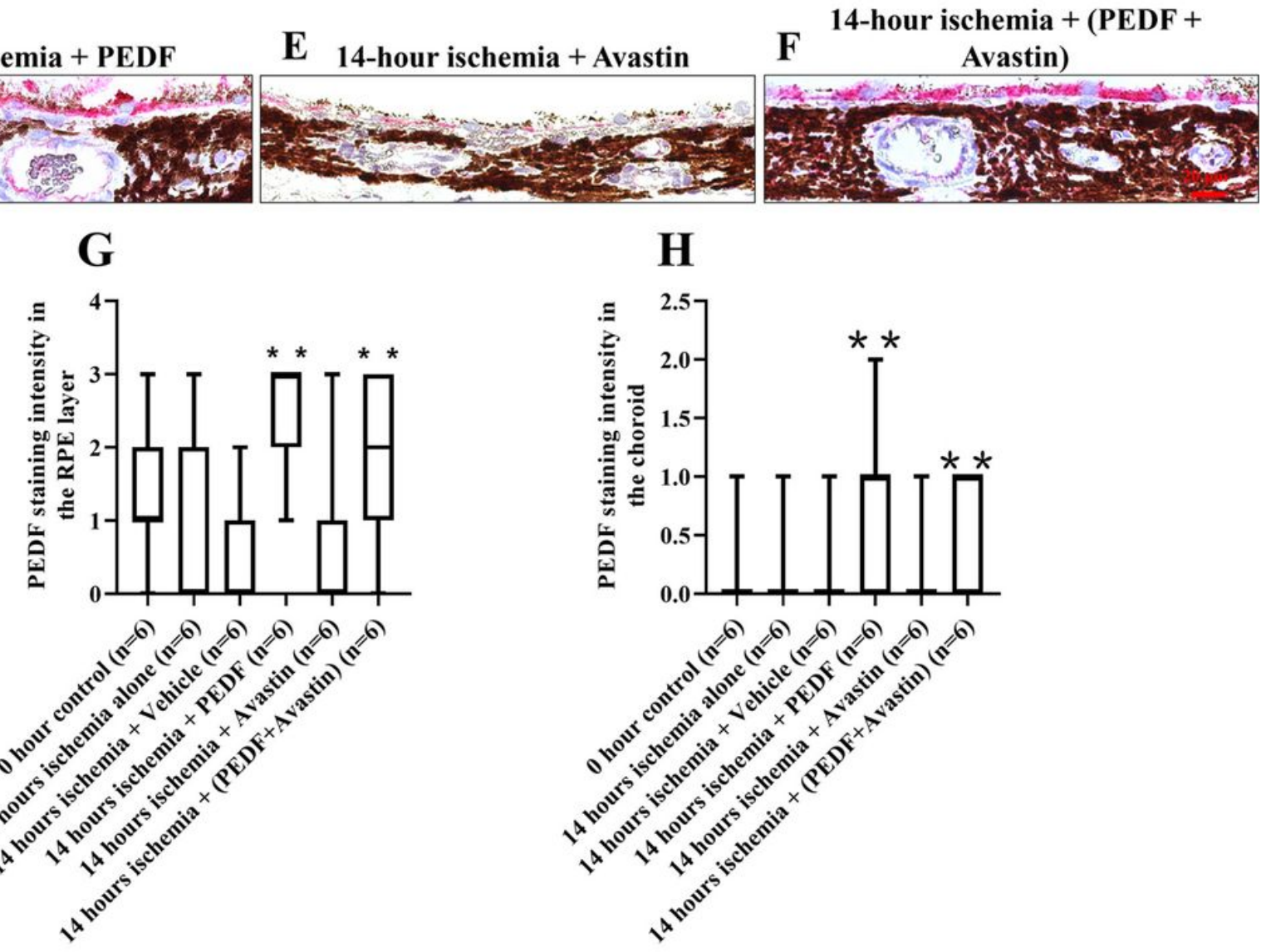

Figure 6

Immunohistochemical staining of PEDF in the RPE cells and the choroid (A-F). The PEDF was detected in the RPE cells and it was nearly negative in the choroid of the 0-hour control group (A), the14 hours hypoxia alone group (B) and the Avastin treatment group (E). The PEDF was nearly negative in the RPE and choroid of the vehicle treatment group (C). The strong immunohistochemical staining in the RPE and choroid vessel walls was found in the PEDF treatment group (D) and the PEDF/Avastin treatment group (F). Immunohistochemical staining intensity of PEDF in the RPE layer $(G)$ and the choroid $(H)$. The PEDF staining intensity was significantly increased in the PEDF treatment group and the PEDF/Avastin treatment group comparing with other groups. Data were analyzed by the Wilcoxon test with KruskalWallis test. ( $\mathbb{\otimes P \otimes 0 . 0 0 1 )}$ (n: number of eyes). RPE: retinal pigmented epithelium. Scale bar $=20 \mu \mathrm{m}$. 

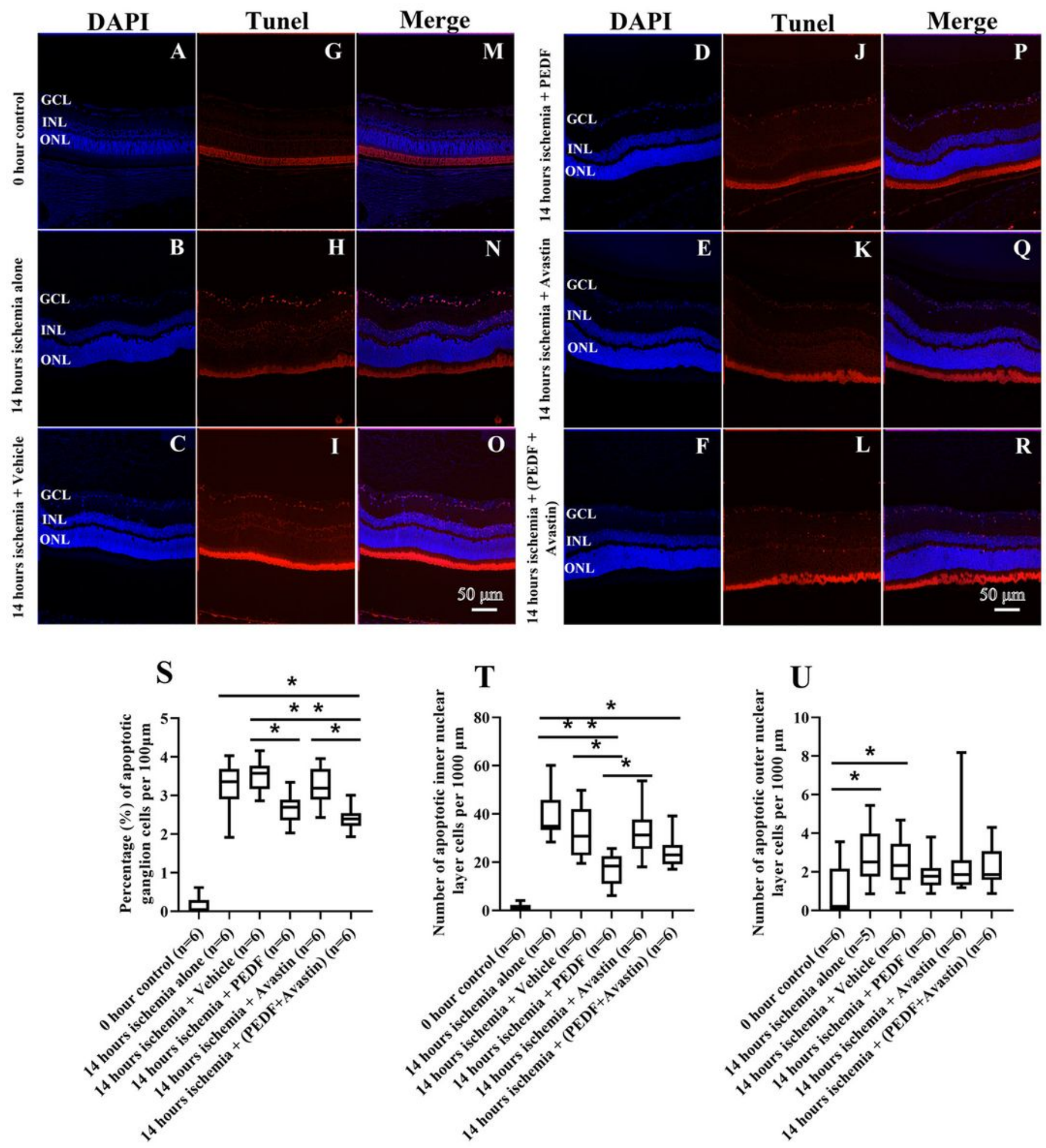

Figure 7

Representative immunofluorescence staining of TUNEL in the central part of the retina, and the analysis of TUNEL-positive cells in the ganglion cells, the inner nuclear layer cells and the outer nuclear layer cells. Figures A-F show the DAPI counterstain, figures G-L show TUNEL-positive staining (red), and figures M-R show the merged TUNEL/DAPI overlay. Positive staining cells were mainly in the inner layers, especially in the GCL. There were hardly any positive staining cells in the control group (M). Following 14 hours of 
ischemia, TUNEL-positive cells (red) were predominantly in the GCL. Some inner nuclear layer cells especially the inner part of the INL were positively stained (H-L). In the PEDF-treated and the PEDF/Avastin-treated groups, some ganglion cells were positively stained and some ganglion cells were negatively stained $(P, R)$. The TUNEL-analysis was performed using the in situ cell death detection TMR red TUNEL kit as recommended by the manufacturer. The percentage of TUNEL-positive nuclei to the total number of ganglion cells per $100 \mu \mathrm{m}$ length of the retina (S). The number of TUNEL-positive nuclei in the inner nuclear layer cells per $1000 \mu \mathrm{m}(\mathrm{T})$. The number of TUNEL-positive nuclei in the outer nuclear layer cells per $1000 \mu \mathrm{m}(\mathrm{U})$. The value is described by percentile (M1, M3) and (Mean $\pm \mathrm{SD})$. Data were analyzed

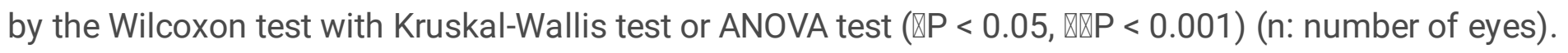
GCL: ganglion cell layer; INL: inner nuclear layer; ONL: outer nuclear layer. Scale bar $=50 \mu \mathrm{m}$. 